\title{
Investigating Methods of Resource Provisioning Mechanisms in Cloud: A Review
}

\author{
Babur Hayat Malik, Talia Anwar, Sadaf Ilyas, Farheen Jafar, Munazza Iftikhar, Maryam Malik, Noreen Islam Deen \\ Department of Computer Science \\ University of Lahore Gujrat \\ Campus, Gujrat
}

\begin{abstract}
Delivering information through cloud computing become a modern computation. For this purpose, electronic device is required to access with an active web server. For delivering different resources, the cloud supplier provides computing power for the cloud users to organize their multiple type of application at any time on different platforms. In cloud computing, the main drawback is relevant to the best use of resources as well as resource provisioning. In cloud computing there is a lack of desired resources that is why the cloud resource provision becomes a daring work. To maintain the quality of services, the provisioning of reasonable resources is need of workloads. The main problem is to find the appropriate workload that depends on the cloud user that is related to resource pair application requirements. This paper reveals the cloud resource provisioning and identification in general and in specific, respectively. In this paper, a methodical analysis of resource provisioning in cloud computing is presented, in which resource provisioning, different types of resource provisioning mechanisms and their comparisons, and benefits are described.
\end{abstract}

Keywords-Resource provisioning; resource provisioning mechanisms; cloud computing; systematic review; comparison between resource provisioning

\section{INTRODUCTION}

In this modern era, cloud computing plays vital role in all types of enterprises [1]. It is also considered as major and famous technology in all institutions and departments such as the different research community, governments, business and education. It also have established its top place in all the activities of the users that depends on the application of cloud computing and in their private lives as well. This medium is getting the most preferable position for presenting the applications that are related to data intensive. By using the strong ways of cloud computing like computation, flexibility, reliability and scalability, we can avoid from the problems and hurdles that creates by big data [2]. We define the cloud computing in the easiest way that provides a platform where the cloud provider store data that is accessible for the end user with the help of internet. The consequence of integrating the cloud computing that consists of multiple functionalities that are delivered to end user via internet by cloud providers, which is one of the cloud services. A software product that the end user use with the help of internet also connected with a web browser on the cloud environment is known as cloud application [3]. The basic technology of cloud computing is virtualization that implies the different operating systems to work on the similar physical type platform and also the structures servers from the Virtual Machines. For delivering multiple resources like storage, CPU, memory, platforms and infrastructure the cloud services provider take help from the Virtual Machine [4].

In the beginning the cloud service providers start to deliver the many kinds of public cloud computing facilities. Cross breed cloud utilized by numerous endeavors and undertakings to build up their own foundation of distributed computing [5]. The main goal of the providers to achieve the strong profit, they deliver the best services and resources to their clients and accommodate with each other. The client in the cloud computing can get and also release different resources by demanding and recurring virtual machine. They have the promptly right for availing the proper and best quality of services not costly at all [6]. The allocation of resources is likely to more difficult as compared to other distributed systems like services of grid computing. The resources of arrangement or management of physical machines can be improved by using different virtual machine. Disk storage, bandwidth, memory and CPU are the multiple physical resources that are attached with virtual machine. Resource utilization is improved and multiplexed the resources in this way [5]. In the form of software application, infrastructure and platform the services are provided. The virtualization technology has become the current progress as computing standard. It represents dynamic provisioning on pay per use basis of computing services. Different pay per use stand services like software as services in information technology industry, platform as a services and infrastructure as services provides by the cloud computing [7]. Table I describes these cloud service models and their description.

TABLE I. Cloud Service Models [8]

\begin{tabular}{|l|l|l|}
\hline Sr\# & Cloud Service Models/Ref & \multicolumn{1}{c|}{ Description } \\
\hline 1 & $\begin{array}{l}\text { Software as a Service } \\
\text { SaaS }\end{array}$ & $\begin{array}{l}\text { Client's side } \\
\text { Managed by a third party vendor } \\
\text { It can be used for conventional } \\
\text { cloud computing applications. }\end{array}$ \\
\hline 2 & $\begin{array}{l}\text { Platform as a Service } \\
\text { PaaS }\end{array}$ & $\begin{array}{l}\text { Development side } \\
\text { Provides a grid or framework } \\
\text { Best use is to develop or } \\
\text { customize the applications. }\end{array}$ \\
\hline 3 & $\begin{array}{l}\text { Infrastructure as a Service } \\
\text { IaaS }\end{array}$ & $\begin{array}{l}\text { Provides a pool of resources of } \\
\text { varied types } \\
\text { Leased by the users according to } \\
\text { their needs and requirements }\end{array}$ \\
\hline
\end{tabular}


Elasticity is the important component of cloud that composes it more and more attempting which also enhance the accessibility of multiple resources in the platform of cloud. Different resource provisioning methods utilized in cloud computing are more attractive if they increase the flexibility of the cloud to the utmost limit. Physical resources in the cloud computing can settle on this limit [9].

\section{RELATED WORK}

All the characteristics and the uses of the cloud resources are under the umbrella of resources arrangement and management [10]. The discovery process is the basic part of resource management. This process includes the finding for the reasonable resource that makes the way with the application requirements. The discovery process is organized from the cloud service provider. The physical computing is delivered by the infrastructure provider [11]. Service provisioning depends on service level agreements, these are the paired of nonfunctional matters and settlement that falls between the clients and service providers. It describes the term for the service that have the qualities of the service i.e. duties, prices and fines if there occurs any kind of violation in agreement. It is important for both the user and cloud services provider the reliable and flexible type of management of the service level agreements. In some cases the avoidance of service level agreements desecration evades the fines that are more costly to the providers. But in some other cases on the origin of timely and flexible responses to the suitable and possible service level agreements like desecration threat, minimum interaction with the system to make the cloud computing more reliable to attain the root and flexible type of on demand computing application. For the assurance of agreement of the service level agreements in the cloud computing, the cloud services provider should be able to observe its infrastructure and also the resource metrics to impel the desired services level purposes and objectives [12].

\section{A. Cloud Workload Management}

The set of examples which about to perform related to the cloud work that is a theoretical work. The effective and legal workload is relevant to running a web, which is one of the examples. In the perspective or framework of the cloud, the project of the workloads is offered. Various cloud workloads and the quality of services demands are not considered by them such as performance, price and time respectively. The users create or generate the workload requests that have been kept on the VI category which should be applied mechanism. Primarily, in the track of the purposes of the cloud users, the cloud users carefully determine that the assigned workload request can be executed. When the workload is admitted, the most important work or project is communicated with the preparations of the parts of the applications which implies the performance that once more lie in the arrangements' objectives of the cloud user. For creating the best and appropriate provisioning system, it is essential to sort or pinpoint the assortment of the cloud workloads and furthermore the nature of the administrations included too [7].

\section{B. Need of Resource Provisioning}

To increase the gratification and the chances or possibility of the users reaching the cloud, there is needed to increase the large number of the requests or feedbacks that gratified from the cloud. Therefore, because of these perspectives the profit becomes so higher to the cloud in the consequence. There is possibility to appeal the customers of the cloud computing application to the cloud is to merge or short time for the responding. To make the attraction of the customers or users with cloud, the cloud is to need for accepting the resource provisioning technique which creates or generates the highest rate of the business deal. For the developing of the higher qualities of the business deal, there is not needed to be settled with the lack of period of the time. By giving the preference to the last, the trade-offs is to be sorted among the transaction success and U-turn time. Hence the shortage of the time can be created as far as it is conceivable for having the main goal to keep the high rate of the dealing success [9].

The applications can be used properly by applying the purpose of resource provisioning which implies that to discover the reasonable resources for the appropriate workloads in time. The best consequences can get by using the more effective resources. The reasonable and appropriate workload discovery is one of the main goals that maintain the program of different workloads. For making the quality of services more effective there is needed to satisfy the parts or units like utility, availability, reliability, time, security, price and CPU etc. So the resource provisioning reflects the performance of the time for the various workloads. All the presentations depend upon the kind or type of workload.

There are entirely two generic way of resource provisioning

- Static Resource Provisioning

- Dynamic Resource Provisioning

\section{Static Resource Provisioning}

For an application all types of desired resources are required in the peak time normally. Mostly this type of cloud provisioning the misuse of resources and wastage of resources because of workload is not considered in the peak time. Despite of this the resource provider offer the maximum desired resource for the purpose of avoids the service level application violation [13].

\section{Dynamic Resource Provisioning}

The customer demand, requirements and workloads are changed rapidly so that the cloud computing contain the elasticity element to the level of advanced automation adaption in the way of resource provisioning. This aim can be achieved through making the automatically scaling up and down of the resources that are assigned to a particular customer. This method is used to match the existing resources with the consumer current needs and demands with more good and reasonable way. In this way the element of elasticity is helpful to overcome the problem of under and over provisioning and also helpful in good and appropriate dynamic resource provisioning [14].

\section{E. Parameters of Resource Provisioning}

1) Response time: The algorithm of resource provisioning is designed to give response in minimum time after completing any task. 
2) Minimize Cost: The cloud services cost should be less for the cloud consumer.

3) Revenue Maximization: The cloud services provider should be earned maximum revenue.

4) Fault tolerant: The algorithm provide services continuously in spite of collapse of nodes.

5) Reduced SLA Violation: The design of algorithm should be capable to decrease SLA violation.

6) Reduced Power Consumption: The placement \& migration methods of virtual machine should be consume low power [13].

\section{RESOURCE PROVISIONING}

The resource provisioning term was commerce in the context of framework and grid computing. Due to lack of required and appropriate resources the cloud resource provisioning becomes a complicated task. In different distributed system the method of resource provisioning frequently contain the objectives and ways of share the workload on different resources and also enhance the amount of resource consumption and also minimize the workload execution time [7]. In cloud application the quality of services contains the provisioning of suitable resources for cloud workload. For the provision of appropriate resources that are used to workloads is a complex work and on the other hand based on quality of services in requirements and also the recognition of suitable resource pair workload in cloud is a hot research issue.

Fig. 1 explains the basic model of resource provisioning in cloud. Cloud user sends their workload like cloud application to the resource provisioning agents and establish good interaction with them. Resource provisioning agent (RPA) does resource provisioning and provide most suitable resource according to the customer requirements. When resource provisioning agent received the workload from user, his connection and access with the resource information centre (RIC) that have all the desired information about all type of resources with a resource pool. After that output can be achieved depend on the workload requirements as précised by consumer. Through resource discovery we know about the available resources and desired resources list can be generated. On the other hand the selection of resources is a procedure of choosing the most appropriate workload resource competition and match depended on the quality of services need expressed by the cloud user in tenure of services level application from the catalog and list which is created by the resource provisioning.

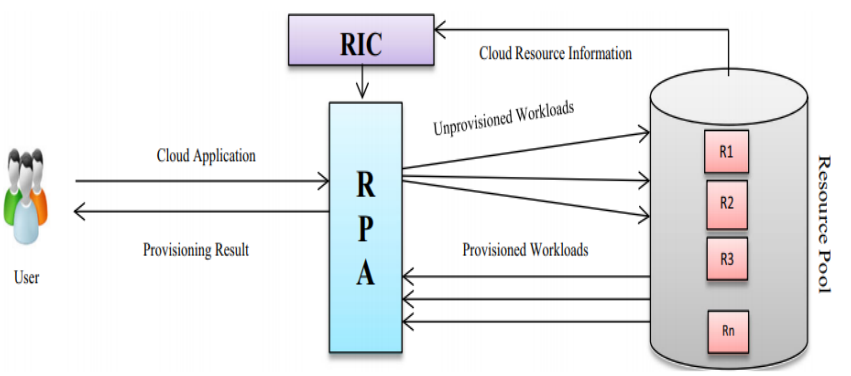

Fig. 1. Basic model of resource provisioning [10].

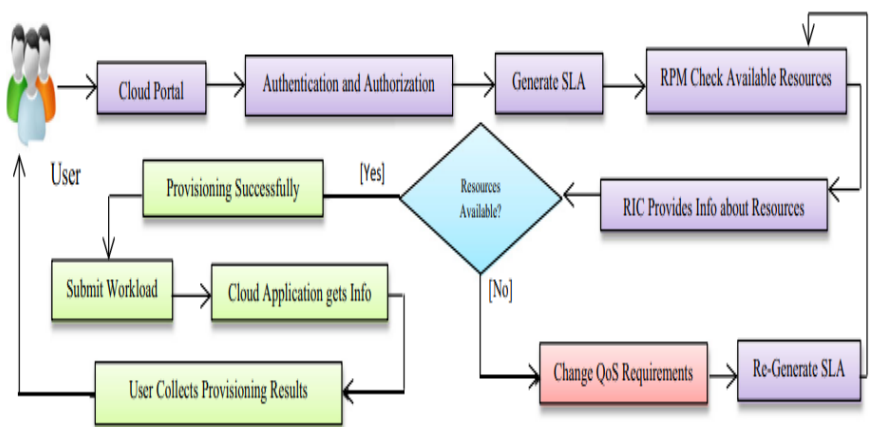

Fig. 2. Flow of resource provisioning [10].

Fig. 2 explains the procedure of cloud resource provisioning. Cloud consumer can interact with the help of cloud portal and also presents the quality of services (QoS) requirements of workload after the complete authentication procedure. Resource information centre (RIC) delivered the information that is based on customer requirements and available resources are checked by the resource provisioning agent (RPA). It is also helpful in provision of desired resources to the cloud application workload for running or execution in the environment of cloud but after fulfill the condition that is demanded resources are present in the resource pool. Resource provisioning agent (RPA) applications for again submit the workload with another quality of services requirement like a service level application article or document in the condition of if the desired resource is unavailable as the requirements of quality of services. Resource scheduler submitted the workloads after the appropriate provisioning of available resources. Resource scheduler requests to submit and present the whole workloads for all the available provisioned resources. Then resource provisioning agent again received the results and send these provisioning outputs and results to the cloud consumer.

\section{Resource PROVISIONING MECHANISEMS}

Some of the extensively and widely used cloud resource discovery and resource provisioning methods or mechanisms are based on the dynamic or distributed resource provisioning. Table II describes some resource provisioning mechanisms.

TABLE II. RESOURCE PROVISIONING MECHANISM

\begin{tabular}{|l|l|l|}
\hline Sr\# & RPM & Description \\
\hline 1 & QoS Based RPM & $\begin{array}{l}\text { The major objective of such work is to } \\
\text { provide provision on different resources } \\
\text { before managing in an appropriate manner } \\
\text { or way and then execute this application } \\
\text { for getting optimal results to the end user. }\end{array}$ \\
\hline 2 & Cost Based RPM & $\begin{array}{l}\text { Minimize the total amount of resource } \\
\text { provisioning cost like over provisioning } \\
\text { cost and under provisioning cost. } \\
\text { Cost reduction can assure the double } \\
\text { capacity of application }\end{array}$ \\
\hline 3 & SLA Based RPM & $\begin{array}{l}\text { SLA provisioning method depend on the } \\
\text { admission control that can be maximizes } \\
\text { the revenue and also the utilization of } \\
\text { resources resource utilization and also pay } \\
\text { attention on multiple type needs of SLA } \\
\text { that are consumer described. }\end{array}$ \\
\hline 4 & Time Based RPM & $\begin{array}{l}\text { Minimum execution time can double the } \\
\text { application capacity as well as minimize } \\
\text { the overhead cost of switching servers. }\end{array}$ \\
\hline
\end{tabular}




\begin{tabular}{|l|l|l|}
\hline 5 & Energy Based RPM & $\begin{array}{l}\text { Enhance the resource utilization and must } \\
\text { be reduce the consumption of power. }\end{array}$ \\
\hline 6 & $\begin{array}{l}\text { Dynamic Based } \\
\text { RPM }\end{array}$ & $\begin{array}{l}\text { envision related to different and changing } \\
\text { user requirements. } \\
\text { Fully and partially distributing the clouds } \\
\text { computing services facilities with other } \\
\text { consumers. }\end{array}$ \\
\hline 7 & $\begin{array}{l}\text { Adaptive Based } \\
\text { RPM }\end{array}$ & $\begin{array}{l}\text { virtualization for the purpose of resource } \\
\text { provisioning depend on the need of } \\
\text { application dynamically as well as } \\
\text { minimize the consumption of power and } \\
\text { energy by maximizing the server's usage. }\end{array}$ \\
\hline 8 & $\begin{array}{l}\text { The running cost of consumer application } \\
\text { can be reduced through advancing the } \\
\text { energy resources and also meet the } \\
\text { required deadline on time make sure that } \\
\text { SLA objectives cannot be violated. }\end{array}$ \\
\hline
\end{tabular}

\section{A. QoS based Resource Provisioning}

The achievement of cloud administrations depends immensely on the level of fulfillment of cloud clients as far as execution and nature of-benefit (QoS) they get from cloud specialist co-ops. QoS alludes to an arrangement of characteristics or qualities of an administration, for example, accessibility, security, reaction time, throughput, inactivity, unwavering quality, and notoriety. Asset provisioning research work in view of QoS has been finished by following creators.

XiaoyongXu et al. [15] propose an occasion driven asset provisioning structure. This system recognizes all occasions that conceivably cause any Map Reduce calculation hard due date absent and pointless asset (Virtual Machine) squander, and instantly handles those occasions. Along these lines, this structure can ensure that the due dates of those Map Reduce calculations running in system are met while limiting the running cost of the structure.

Bahman Javadi et al. [16] considered the issue of QoSbased asset provisioning in a cross breed Cloud figuring framework where the private Cloud is disappointment inclined and built up an adaptable and versatile half breed Cloud design to take care of the issue of asset provisioning for clients' solicitations. The proposed engineering uses the Inter Grid ideas which depend on the virtualization innovation and embrace an entryway (IGG) to interconnect distinctive asset suppliers. The creator proposed facilitating techniques in the half breed Cloud framework where an association that works its private Cloud plans to enhance the QoS for the clients' solicitations by using the general population Cloud assets.

Sukhpal Singh Gill et al. [17] show a keen QoS-mindful autonomic asset administration approach named as CHOPPER (Configuring, Healing, Optimizing and Protecting Policy for Efficient Resource administration). CHOPPER offers selfsetup of uses and assets, self-mending by taking care of sudden disappointments, self-protection against security assaults and self-advancement for greatest asset utilization. Author assessed the execution of the proposed approach in a genuine cloud condition and the trial comes about show that the proposed approach performs better as far as cost, execution time, SLA infringement, asset dispute and furthermore gives security against assaults.

\section{B. Cost based Resource Provisioning}

Cost provisioning research work has been finished by following creators.

Aarti Singh et al. [7] proposes another Agent based Automated Service Composition (A2SC) calculation containing demand handling and computerized benefit organization stages and isn't in charge of seeking thorough administrations yet in addition considers lessening the cost of virtual machines which are devoured by on-request benefits as it were.

Adel Nadjaran Toosi et al. [18] propose another asset provisioning calculation to help the due date prerequisites of information escalated applications in crossover cloud situations. To assess this proposed calculation, creator actualizes it in Aneka, a stage for creating adaptable applications on the Cloud. Trial comes about utilizing a genuine contextual analysis executing an information concentrated application to quantify the walk capacity list on a half and half cloud stage comprising of dynamic assets from the Microsoft Azure cloud demonstrate that the proposed provisioning calculation can all the more productively dispense assets contrasted with existing techniques.

SmitaVijayakumar et al. [19] consider versatile spilling applications where a client needs to accomplish the base asset costs while keeping up a predetermined exactness objective. Creator shows a dynamic and robotized system which can adjust the versatile parameters to meet the particular exactness objective, and afterward powerfully focalize to close ideal asset distribution. This arrangement can deal with surprising changes in the information appropriation qualities and additionally rates. Creator assesses our approach utilizing two gushing applications and exhibits the adequacy of our structure.

Safiye Ghasemi et al. [2] proposed a novel learning based asset provisioning approach that accomplishes cost-decrease certifications of requests. The commitments of this upgraded asset provisioning (ORP) approach are as per the following. Right off the bat, it is intended to give a financially savvy strategy to proficiently deal with the provisioning of asked for applications. ORP performs in light of administrations of which applications included and thinks about their proficient provisioning completely. Furthermore, it is a learning automata-based approach which chooses the most appropriate assets for facilitating each administration of the requested application. Thirdly, a far reaching assessment is performed for three regular workloads: information escalated, processconcentrated and ordinary applications.

\section{SLA based Resource Provisioning}

Distributed computing depends on getting to each sort of asset through an "as-a-benefit" interface, and on the reception of a compensation for every utilization plan of action. In such a specific situation, Security Service Level Agreements (SLAs) expect a key part, as they permit, in addition to other things, to announce plainly the security level conceded by suppliers to clients, and also the imperatives postured to the two gatherings (suppliers and customers).

Yoori Oh et al. [20] propose an auto-scaling system with relating calculations to oversee assets powerfully in virtual 
conditions, so as to meet client determined SLA (Service Level Agreement) given an arrangement of restricted assets. In this paper, he proposes an auto-scaling strategy for using asset of Spark groups successfully in distributed computing condition. The proposed auto-scaling technique has an objective to meet client indicated due date. Likewise perform tests to check the adequacy of the proposed scaling calculation.

Elarbi Badidi et al. [21] propose a system for SaaS provisioning, which depends on expedited Service Level assentions (SLAs), between benefit buyers and SaaS suppliers. A Cloud Service Broker (CSB) helps shoppers choosing the privilege SaaS supplier that can satisfy their useful and nature of-benefit (QoS) prerequisites. Besides, the CSB is responsible for arranging the SLA expressions utilizing a multicharacteristics transaction display with a chose SaaS supplier in the interest of the administration purchaser, and checking the consistence to the SLA amid its usage.

Obinna Anya et al. [22] present an approach for versatile administration provisioning in the Cloud in view of QoS examination. A noteworthy commitment of the approach is the improvement of an examination motor for prescient flexibility administration of Cloud benefit provisioning that incorporates top to bottom mining of SLA consistence history with learning of business setting, e.g. workload fluctuation, a client's business objectives, application execution, and administration operational setting. In this work-in-advance report, creator portrays the proposed system and talks about conceivable usage and arrangement situations.

Valentina Casola et al. [23] introduce the SPECS system, which empowers the improvement of secure cloud applications secured by a Security SLA. The SPECS structure offers APIs to deal with the entire Security SLA life cycle and gives every one of the functionalities expected to automatism the implementation of appropriate security systems and to screen client characterized security highlights. The improvement procedure of SPECS applications offering security-upgraded administrations is represented, exhibiting as a certifiable contextual investigation the provisioning of a safe web server.

\section{Time based Resource Provisioning}

Time provisioning research work has been finished by following creators.

Lakshmi Ramachandran et al. [24] propose a novel User Interface-Tenant Selector-Customizer (UTC) model and approach, which empowers cloud-based administrations to be methodically demonstrated and provisioned as variations of existing administration occupants in the cloud. This approach thinks about utilitarian, non-practical and asset allotment prerequisites, which are unequivocally indicated by the customer through the UI segment of the model. This is the primary such coordinated approach that represents the thoughts utilizing a practical running case, and furthermore exhibit a proof-of-idea model manufactured utilizing IBM's Rational Software Architect displaying device.

Izzet F. Senturk et al. [25] propose BioCloud as a solitary purpose of passage to a multi-cloud condition for non-PC adroit bio analysts. They talk about the design and segments of BioCloud and present the planning calculation utilized in
BioCloud. Trials with various utilize cases and situations uncover that BioCloud can diminish the worked execution time for a given spending plan while typifying the multifaceted nature of asset administration in numerous cloud suppliers.

\section{E. Energy based Resource Provisioning}

Regardless of the achievement of some outstanding CSPs, for example, Google App Engine (GAE) and Amazon Elastic Compute Cloud (EC2), the huge vitality costs as far as power devoured by server farms is a genuine test. Vitality use of server farms has two critical highlights: (i) servers have a tendency to be more vitality wasteful under low usage rate, and (ii) servers may expend a lot of energy out of gear mode.

YaGao et al. [26] create measurable nature of administration (QoS) driven power control approaches to expand the successful vitality proficiency (EEE), which is characterized as the range effectiveness under given determined QoS imperatives per unit reaped vitality, for vitality gathering based remote systems. Specifically, to begin with, break down the long haul accessible vitality imperatives and detail the EEE amplification issue. At that point, determine the shut frame arrangements of ideal power control strategies to the EEE augmentation issue under the battery limit over whelmed imperative, the normal collected vitality requirement, and both the battery limit and normal gathered vitality limitations, individually.

Mingxi Cheng et al. [4] presents DRL-Cloud, a novel Deep Reinforcement Learning (DRL)- based RP and TS framework, to limit vitality cost for vast scale CSPs with substantial number of servers that get colossal quantities of client demands every day. A profound Q-learning-based two-organize RP-TS processor is intended to naturally produce the best long haul choices by gaining from the changing condition, for example, client ask for designs and reasonable electric cost. With preparing strategies, for example, target arrange, encounter replay, and investigation and misuse, the proposed DRL-Cloud accomplishes astoundingly high vitality cost productivity; low reject rate and in addition low runtime with quick merging. Contrasted and one of the best in class vitality effective calculations, the proposed DRL-Cloud accomplishes up to $320 \%$ vitality cost proficiency change while keeping up bring down reject rate all things considered.

\section{F. Dynamic based Resource Provisioning}

The one element of cloud that makes it speaking to its clients is Elasticity which expands the accessibility of assets in the cloud. The asset provisioning strategy utilized as a part of a cloud is said to be sound in the event that it improves the cloud's versatility to as far as possible. This point of confinement is controlled by the measure of physical assets in the cloud.

Kirthica S. et al. [9] give a proposition. That proposition means to supplant the existing asset provisioning strategy in an open system, Cloud Inter-task Toolkit (CIT), to make an expanded exchange progress rate which is apparent from the experimental comes about acquired from an ongoing heterogeneous cloud condition set up utilizing Eucalyptus, OpenNebula and OpenStack. Notwithstanding fulfilling a demand with assets from numerous mists, these assets are 
totaled and given in a way that is effectively accessible by the client.

\section{G. Adaptive based Resource Provisioning}

It is trying for cloud suppliers to assign the pooled processing assets progressively among the separated clients as to amplify their income. It isn't a simple errand to change the client arranged administration measurements in to working level measurements, and control the cloud assets adaptively in light of Service Level Agreement (SLA) [27].

Guofu Feng et al. [27] addresses the issue of amplifying the supplier's income through SLA-based dynamic asset designation as SLA assumes an essential part in distributed computing to connect specialist organizations and clients. Creator formalizes the asset portion issue thinking about different Quality of Service (QoS) parameters.

Ayoub Alsarhan et al. [28] propose a novel Service Level Agreement (SLA) structure for distributed computing, in which a value control parameter is utilized to meet QoS requests for all classes in the market. The structure utilizes fortification learning (RL) to determine a VM procuring arrangement that can adjust to changes in the framework to ensure the QoS for all customer classes. These progressions include: benefit cost, framework limit, and the interest for benefit. This approach incorporates processing assets adjustment with benefit confirmation control in light of the RL show.

Abiola Adegboyega et al. [29] built up a model to anticipate transfer speed usage pertinent in keeping up SLAs for various activity streams at the cloud organize edge and center. The created univariate estimate show utilizes the AutoRegressive Integrated Moving Average (ARIMA) demonstrate expanded with a general class of Adaptive Conditional Score Models (ACS). Creator inspiration for utilizing the ACS comes from its powerful adjustment to exceptions and drifters more proficiently with expanded computational exactness than current strategies; one of such techniques being the as of late embraced Generalized Auto-Regressive Conditional Heteroskedasticity (GARCH) to show instability.

\section{H. Optimization based Resource Provisioning}

Enhancing provisioning research work has been done by following makers.

YaGao et al. [26] create factual nature of administration (QoS) driven power control strategies to augment the compelling vitality effectiveness (EEE), which is characterized as the range proficiency under given indicated QoS limitations per unit collected vitality, for vitality gathering based remote systems. Specifically, in the first place, creator breaks down the long haul accessible vitality imperatives and defines the EEE augmentation issue. At that point, infer the shut frame arrangements of ideal power control strategies to the EEE boost issue under the battery limit overwhelmed imperative, the normal collected vitality limitation, and both the battery limit and normal gathered vitality requirements, individually.

Marcus Lemos et al. [30] ACOSIM, an approach ACOSIM, to limit the general sensor cloud vitality utilization by choosing just a subset of sensor hubs to create the virtual sensors. Results from starting investigations demonstrate that the approach decreases the sensor cloud vitality utilization the by $73.97 \%$, giving an answer for be considered in sensor cloud situations.

\section{COMPARISION OF RESOURCE PROVISIONING MECHANISEMS}

Examination of asset provisioning systems is a troublesome assignment because of various sorts of asset provisioning components and the absence of benchmarks. We considered distinctive characteristics of asset provisioning components and look at them.

\section{A. Traits of Resource Provisioning}

RPM in cloud frameworks can be looked at in view of some normal qualities for taking care of provisioning issues. Searching mechanism, objective function, resource provisioning strategy, merits and demerits are a portion of the normal and essential qualities that ought to be inspected in every RPM as depicted in Table III. Table IV demonstrates the difference of asset provisioning components in view of these qualities.

TABLE III. RESOURCE PROVISIONING TRAITS

\begin{tabular}{|c|l|l|}
\hline Sr\# & \multicolumn{1}{|c|}{ Traits } & \multicolumn{1}{|c|}{ Description } \\
\hline 1 & Provisioning Mechanism & Strategy for give assets \\
\hline 2 & Searching Mechanism & $\begin{array}{l}\text { Finding the best workloads and assets } \\
\text { relies upon seeking speed.. }\end{array}$ \\
\hline 3 & Objective Function & $\begin{array}{l}\text { A target capacity of each RPM is } \\
\text { particularly intended for a particular } \\
\text { reason for the system.. }\end{array}$ \\
\hline 4 & $\begin{array}{l}\text { Resource Provisioning } \\
\text { Strategy }\end{array}$ & $\begin{array}{l}\text { The methodology of giving assets to } \\
\text { workloads execution is called Resource } \\
\text { Provisioning Strategy (RPS) scientific } \\
\text { classification. Two kinds of RPS scientific } \\
\text { classification are depicted beneath: } \\
\text { - Dynamic } \\
\text { - Distributed }\end{array}$ \\
\hline 5 & Merits & $\begin{array}{l}\text { The benefits of Resource Provisioning } \\
\text { Mechanismare depicted in this segment. }\end{array}$ \\
\hline 6 & Demerits & $\begin{array}{l}\text { The disservices of Resource Provisioning } \\
\text { Mechanismare depicted in this segment. }\end{array}$ \\
\hline
\end{tabular}

B. Resource Provisioning Comparison

TABLE IV. RESOURCE PROVISIONING COMPARISON

\begin{tabular}{|c|c|c|c|c|c|c|c|}
\hline $\begin{array}{l}\mathbf{S} \\
\mathbf{r} \\
\#\end{array}$ & $\begin{array}{c}\text { Provi } \\
\text { sioni } \\
\text { ng } \\
\text { Mec } \\
\text { hanis } \\
\text { m/Re } \\
\text { f }\end{array}$ & Topic & $\begin{array}{c}\text { Searching } \\
\text { Mechanis } \\
\text { m }\end{array}$ & $\begin{array}{l}\text { Objective } \\
\text { function }\end{array}$ & RPS & Merits & $\begin{array}{c}\text { Deme } \\
\text { rits }\end{array}$ \\
\hline 1 & $\begin{array}{l}\text { Cost } \\
\text { Base } \\
\mathrm{d} \\
\text { RPM } \\
/[30][ \\
19]\end{array}$ & $\begin{array}{l}\text { Compe } \\
\text { tent } \\
\text { resourc } \\
\text { e } \\
\text { provisi } \\
\text { oning } \\
\text { and } \\
\text { distribu } \\
\text { tion } \\
\text { techniq } \\
\text { ues } \\
\text { for } \\
\text { cloud }\end{array}$ & $\begin{array}{l}\text { KFCM } \\
\text { algorithm } \\
\text { was used } \\
\text { to cluster } \\
\text { the } \\
\text { available } \\
\text { resource.Pa } \\
\text { rticle } \\
\text { swarm } \\
\text { optimizatio } \\
\text { n algorithm } \\
\text { is used to } \\
\text { select the }\end{array}$ & $\begin{array}{l}\text { To } \\
\text { distribute } \\
\text { the } \\
\text { resourcesi } \\
\text { n- an } \\
\text { powerful } \\
\text { way }\end{array}$ & $\begin{array}{l}\text { Distr } \\
\text { ibuti } \\
\text { ve }\end{array}$ & $\begin{array}{l}\text { This } \\
\text { system } \\
\text { accomp } \\
\text { lishes } \\
\text { least } \\
\text { executi } \\
\text { on } \\
\text { time, } \\
\text { least } \\
\text { cost } \\
\text { esteem } \\
\text { and } \\
\text { low }\end{array}$ & $\begin{array}{l}\text { Calcul } \\
\text { ation } \\
\text { Diffic } \\
\text { ult }\end{array}$ \\
\hline
\end{tabular}




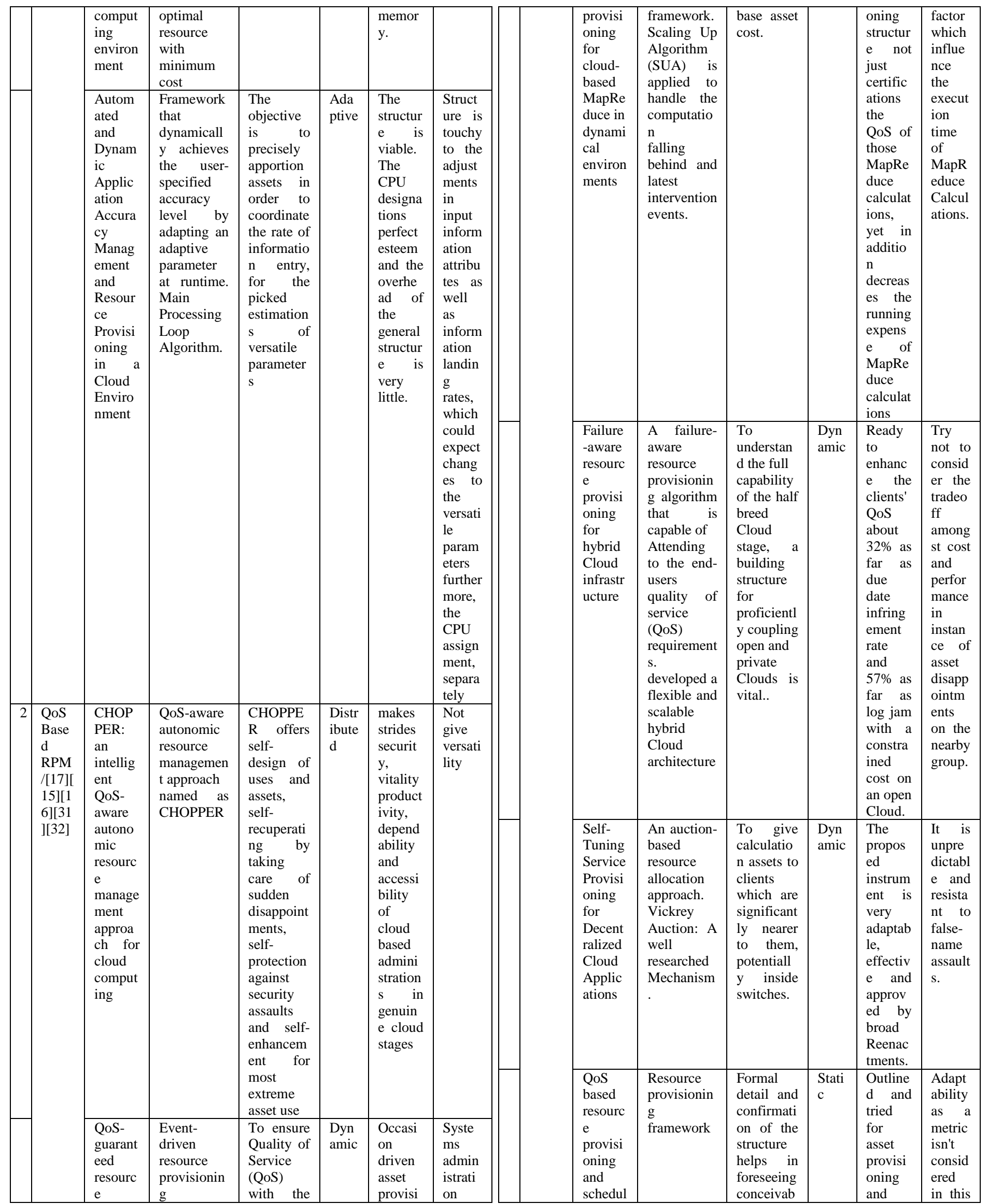




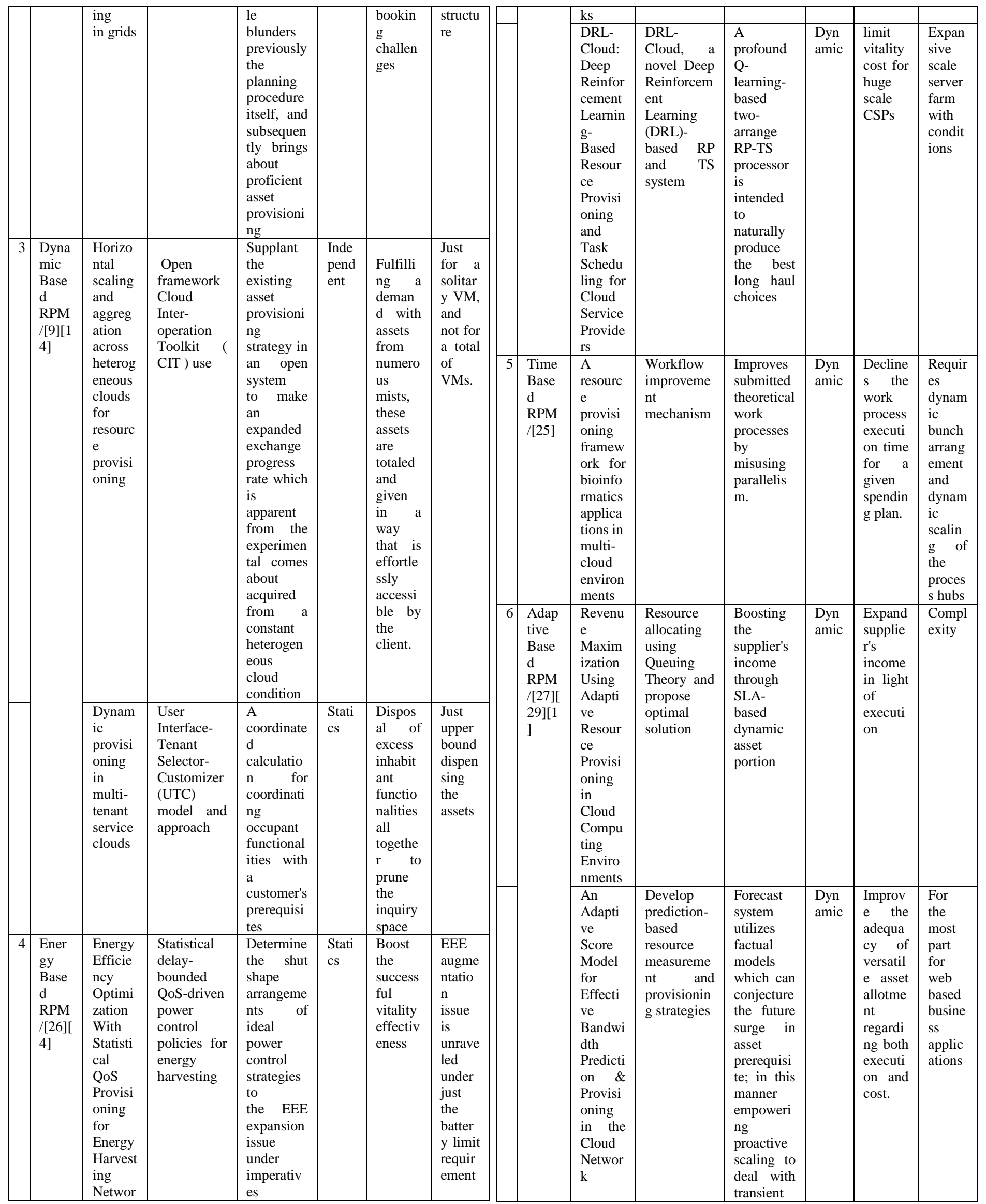




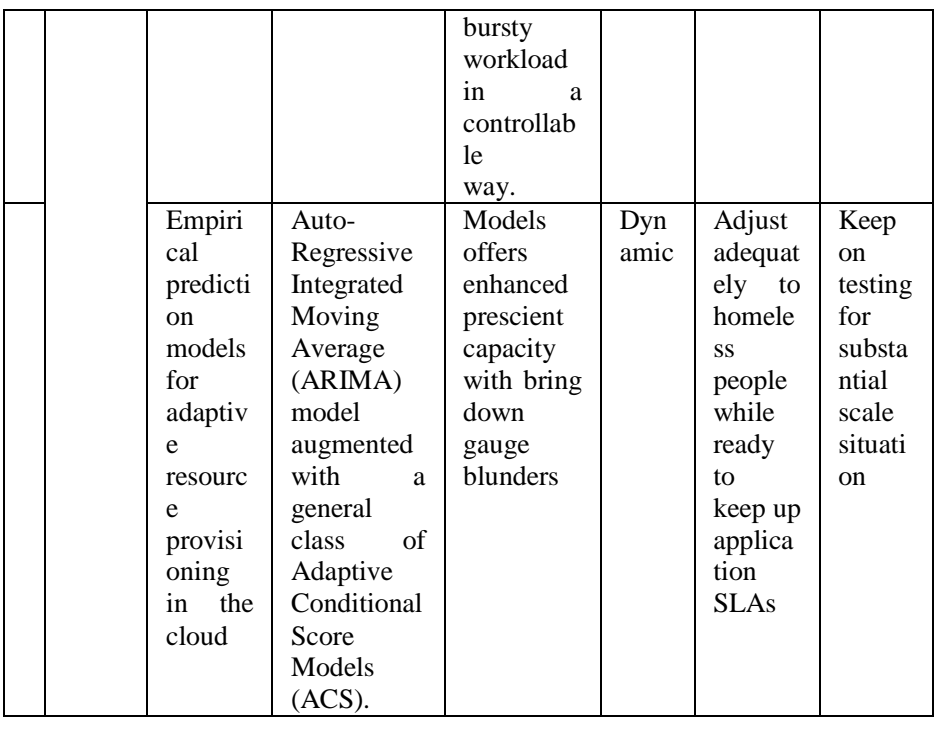

\section{Benefits of Resource Provisioning}

- Effective cloud asset provisioning lessens execution time of cloud workloads.

- Better asset usage under various prerequisites of need and maintains a strategic distance from over provisioning and under provisioning.

- No provisioning delay and lesser odds of asset disappointment because of productive administration of assets.

- No long VM start-up delay gives provisioned assets promptly in compelling cloud asset provisioning.

- Increase the vigor and limit make traverse of work process at the same time.

- Meet even strict application due date with least spending consumption and increments worldwide benefit.

- Power utilization lessened without infringement of SLA in powerful cloud asset provisioning.

- Efficient adjusting of load by proficient dissemination of the workloads on accessible assets.

- Improve client due date infringement rate because of assets provisioning before asset planning.

- Effective cloud asset provisioning decreases lining time in workload line.

\section{CONCLUSION}

The examinations which were contemplated above are attempting to improve and use the assets. A few techniques were said here which utilized diverse parameters as an objective for asset provisioning, for example, reaction time, dismissal rate, benefit level assention (SAL) infringement rate, cost and so forth. For provisioning arranging should take proper provisioning times, Provisioning assets too early will squanders our assets and in this way our cash, on the opposite side provisioning assets past the point of no return will cause possibly SLA infringement and makes the clients furious. This paper displays an exhaustive audit on successful assets provisioning in cloud. We have talked about asset provisioning when all is said in done. We outlined asset provisioning component and correlation between various assets provisioning instrument as far as a superior execution, focused and productivity to meet the required SLA enhanced the asset execution and brought down the power utilization. So we reason that portion of assets in light of kind of workload. Appropriate coordinating of workload and asset can enhance the execution significantly. It is extremely troublesome for supplier to recognize the quantity of assets required precisely for given workload from asset pool, since assets might vary in one or other criteria for example, asset limit, cost and speed. User can choose fitting asset provisioning component based on QoS necessities of workload/application portrayed through assessment and correlation of asset provisioning in cloud. Differentiation and evaluation of asset provisioning systems in cloud can help to choose the asset provisioning instrument in view of workload's QoS requirements. Cost can be lessened in the conveyed cloud benefit if assets are held ahead of time. We trust this paper will spur specialists to investigate and figure another system to explain issues in designating and checking assets in distributed computing and this research work will be beneficial for researchers who want to do research in area concerning to resource management such as cloud resource provisioning and resource provisioning mechanism.

\section{REFERENCES}

[1] "Empirical prediction models for adaptive resource provisioning in the cloud", Sadeka Islam and et al. 2011.

[2] "A cost-aware mechanism for optimized resource provisioning in cloud computing", Safiye Ghasemi and et al.2017.

[3] "An autonomic resource provisioning approach for service-based cloud applications: A hybrid approach", Mostafa Ghobaei-Arani and et al. 2017.

[4] "DRL-Cloud: Deep Reinforcement Learning-Based Resource Provisioning and Task Scheduling for Cloud Service Providers", Mingxi Cheng and et al. 2018.

[5] "Resource Provision Algorithms in Cloud Computing: A Survey", Jiangtao Zhang and et al. 2016.

[6] "Combinatorial Double Auction-based Resource Allocation Mechanism in Cloud Computing Market", Seyedeh Aso Tafsiri and et al. 2017.

[7] "A Novel Agent Based Autonomous and Service Composition Framework for Cost Optimization of Resource Provisioning in Cloud Computing", Aarti Singh and et al. 2015.

[8] "Review on: Resource Provisioning in Cloud Computing Environment", Sagar Girase and et al. 2013.

[9] "Horizontal scaling and aggregation across heterogeneous clouds for resource provisioning", Kirthica S and et al. 2017.

[10] "Cloud resource provisioning: survey, status and future research directions", Sukhpal Singh and et al. 2016.

[11] "A Survey on Resource Allocation and Monitoring in Cloud Computing", Mohd Hairy Mohamaddiah and et al. 2014.

[12] "Cloud resource provisioning and SLA enforcement via LoM2HiS framework", Vincent C. Emeakaroha and et al. 2012.

[13] “ Resource Provisioning in Single Tier and Multi-Tier Cloud Computing: - State-of-the-Art", Marwah Hashim Eawna and et al. 2015.

[14] "Multi-agent based dynamic resource provisioning and monitoring for cloud computing systems infrastructure", Mahmoud Al-Ayyoub and et al. 2015.

[15] "QoS-guaranteed resource provisioning for cloud-based MapReduce in dynamical environments", Xiaoyong Xu and et al. 2018. 
[16] "Failure-aware resource provisioning for hybrid Cloud infrastructure", Bahman Javadi and et al. 2012.

[17] "CHOPPER: an intelligent QoS-aware autonomic resource management approach for cloud computing", Sukhpal Singh Gill and et al. 2017.

[18] "Resource provisioning for data-intensive applications with deadline constraints on hybrid clouds using Aneka", Adel Nadjaran Toosi and et al. 2017.

[19] "Automated and Dynamic Application Accuracy Management and Resource Provisioning in a Cloud Environment", Smita Vijayakumar and et al. 2010.

[20] "A SLA-based Spark Cluster Scaling Method in Cloud Environment", Yoori Oh and et al. 2016.

[21] "A Cloud Service Broker for SLA-based SaaS Provisioning", Elarbi Badidi. 2013.

[22] "SLA Analytics for Adaptive Service Provisioning in the Cloud", Obinna Anya and et al. 2016.

[23] "SLA-based Secure Cloud Application Development: the SPECS Framework", Valentina Casola and et al. 2016.
[24] "Dynamic provisioning in multi-tenant service clouds", Lakshmi Ramachandran and et al. 2012.

[25] "BioCloud: A resource provisioning framework for bioinformatics applications in multi-cloud environments", Izzet F. Senturk and et al. 2018.

[26] "Energy Efficiency Optimization With Statistical QoS Provisioning for Energy Harvesting Networks", Ya Gao and et al. 2017.

[27] "Revenue Maximization Using Adaptive Resource Provisioning in Cloud Computing Environments", Guofu Feng and et al. 2012.

[28] "Adaptive Resource Allocation and Provisioning in Multi-Service Cloud Environments", Ayoub Alsarhan and et al. 2017.

[29] "An Adaptive Score Model for Effective Bandwidth Prediction \& Provisioning in the Cloud Network", Abiola Adegboyega. 2015.

[30] "An Algorithm Based On Ant Colony Optimization for Provisioning Virtual Sensor in Sensor Cloud", Marcus Lemos and et al. 2017.

[31] "Self-Tuning Service Provisioning for Decentralized Cloud Applications", Raul Landa and et al. 2013

[32] "QoS based resource provisioning and scheduling in grids", Rajni Aron and et al. 2013 